Military Technical College Kobry El-Kobba

Cairo, Egypt

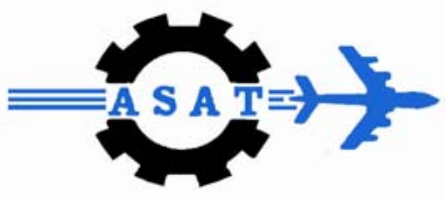

12-th International Conference

on

Aerospace Sciences \&

Aviation Technology

\title{
INVESTIGATING A FRONTIER OF TLM TO ANALYZE FSS
}

\author{
ABD-ALAZIZ A.M., MOHAMMED H.Z., HOSSAM H. *
}

\begin{abstract}
:
The Transmission Line Matrix Method (TLM) is based on a discretization criterion. The paper discusses the limit of relative discretization length (with respect to wavelength) to accurately represent planar structures using the TLM. Convergence of the solution of the TLM with this limit is discussed in an analysis of Frequency Selective Surfaces (FSS) by changing its parameters (shape, dimensions and number of layers).

Perfect matching between the TLM and the MoM results is achieved, in addition to the measured results of some fabricated structures.
\end{abstract}

\section{KEY WORDS:}

Transmission Line Matrix Method, Relative discretization length, Frequency Selective Surfaces. 


\section{* Egyptian Armed Forces}

\section{I- Introduction to Transmission Line Matrix method:}

The Transmission Line Matrix method is a physical model to represent the electric and magnetic fields in Maxwell's equation by the corresponding voltages and currents on a transmission line which is descritized into sections of relative length $(\Delta l / \lambda)$.

Based on John's equation:

$$
\frac{\beta}{\beta_{n}}=\frac{\pi\left(\frac{\Delta l}{\lambda}\right)}{\sin ^{-1}\left[2 \sin \left(\frac{\pi \Delta l}{\lambda}\right)\right]}
$$

where, $\beta_{\mathrm{n}}$ is phase constant in the TLM network and $\beta$ is phase constant in free space, the wave velocity in the 3D-Symmetrical Condensed Node (SCN) TLM network is one half of that in free space. In order to represent the wave propagation correctly (for lossless media), the corresponding transmission line should has $(\Delta \mathrm{l} / \lambda)$ not exceeding $0.166 \dot{6}[1]$. This value is an upper limit for using this method. The TLM is not valid for higher $(\Delta l / \lambda)$ because the solution is no longer exists as illustrated in figure (1).

One field of application of the TLM is the analysis of Frequency Selective Surfaces (FSS). The FSS are periodic structures, either metallic patches on a dielectric substrate (capacitive type) or perforated apertures on conducting sheet (inductive type), which perform a filter operation for electromagnetic waves [2].

The importance of FSS is simply the shielding against EM radiation (in case of capacitive structure) since it provides zero transmission at the resonance frequency, while its complement (inductive type) provides zero reflectivity at the resonance frequency and can be used to absorb EM radiation which makes the target invisible for radiation.

In this work, infinite FSS structures are analyzed using TLM and the attained results are compared with the results of the MoM (using ANSOFT DESIGNER software) [3], in addition to some experimental results.

The paper is organized as follows; Section I is the introduction section. The application of the TLM in the analysis of FSS is described in Section II. The numerical results and measurements are presented in Section III. Finally the conclusion is summarized in section IV.

\section{II-The TLM and its Frontier in the Analysis of FSS:}

To analyze an infinite FSS structure using TLM (figure 2-a), a perfect electric (short circuit) and magnetic walls (open circuit) are introduced at the sides of the structure. The perfectly reflecting walls act as mirrors to simulate the rest of the infinite structure, so only one quarter of the cross dipole will be introduced (Figure 2-b)[5]. 
In order to ensure the propagation in TLM structure in one way (traveling wave), a Perfect Matched Layers (PMLs) are introduced in front of and at the end of the structure to ensure the appropriate propagation (Figure 2-c).

The reflection coefficient can be then calculated using conservation of energy by subtracting the transmitted field form the incident one.

Many examples were held to investigate the appropriate $(\Delta I / \lambda)$ experimentally by simulating FSS structures, and it was found that the convergence of TLM solution happens as $(\Delta \mathrm{l} / \lambda)$ approaches 0.016 .

Figure (3) shows the results for a rectangular loop capacitive type FSS which was fabricated and experimentally measured to resonate at $(10.73 \mathrm{GHz})$ while the MoM showed a resonance at $(10.75 \mathrm{GHz})$. As for TLM, $(\Delta \mathrm{l} / \lambda)$ approaching $(0.0148)$ the resonance occurs at $(10.745 \mathrm{GHz})$. Increasing $(\Delta I / \lambda)$ to be about $(0.027)$ shifts the resonance frequency about (4.15\%) from the original one, which is a drawback for this selection. More increasing of $(\Delta I / \lambda)$ shows worse result far away from the actual one, although all these values still nearly in the linear part of John's curve.

Figure (4) is the results for a crossed dipole FSS which resonates at $(10.25 \mathrm{GHz})$ (MoM result). The figure shows good matching between the MoM and TLM specified $(\Delta \mathrm{l} / \lambda)$ while other results are far from the right one.

Figure (5) shows, for a Jerusalem crossed dipole, good matching with $(\Delta \mathrm{l} / \lambda)$ equals to $(0.009)$ and a slight shift in resonance frequency for $(\Delta l / \lambda)$ equals to $(0.0178)$.

\section{Numerical Results and Measurements}

This section presents the analysis of crossed dipole FSS using TLM. The crossed dipole element is a simple and promising FSS structure (consists of two orthogonal low and high pass filters forming a band stop one). Also it has the advantage of polarization independency because of its symmetrical shape.

The simulations apply $(\Delta I / \lambda)$ equals to 0.016 to ensure the stability of the method. The iteration process was done for 20,000 times at least to ensure steady state solutions. The structure has a single layer with $L=12 \mathrm{~mm}, a=4 \mathrm{~mm}$ and $b=2.4 \mathrm{~mm}$ Simulations and measurement in figure (6) show the agreement of TLM result with the other results.

The following figures exhibit the flexibility and accuracy of the TLM method in studying the effect of different FSS geometrical configurations and a study to the bandwidth of FSS structure $[5,6]$. Changing the element length (L) changes the resonance frequency (figure 7); according to the empirical formula in [7]:

$\lambda_{r}=2.27(L+a)-b-2 a$

where $\lambda_{r}$ is the resonance wavelength.

Other parameters such as changing the element width changes the bandwidth with a slight change in frequency (according to equation 2) as shown in figure (8), table(1).

Table 1: Comparison of Bandwidth from changing width point of view

\begin{tabular}{|c|c|c|c|}
\hline Simulation method & $b=2.4 \mathrm{~mm}$ & $b=4 \mathrm{~mm}$ & $b=5.6 \mathrm{~mm}$ \\
\hline
\end{tabular}




\begin{tabular}{|c|c|c|c|}
\hline TLM & $13 \%$ & $17.9 \%$ & $21 \%$ \\
\hline MOM & $13.07 \%$ & $19.6 \%$ & $24 \%$ \\
\hline
\end{tabular}

Figure (9) shows that decreasing the spacing between the FSS elements increase the band width and summarized in table (2).

Table 2: Comparison of BW from spacing point of view

\begin{tabular}{|c|c|c|c|}
\hline Simulation method & $\mathrm{a}=2.4 \mathrm{~mm}$ & $\mathrm{a}=3.2 \mathrm{~mm}$ & $\mathrm{a}=4 \mathrm{~mm}$ \\
\hline TLM & $17.94 \%$ & $16.5 \%$ & $13.15 \%$ \\
\hline MOM & $19.8 \%$ & $16.88 \%$ & $14.47 \%$ \\
\hline
\end{tabular}

Figure 10, table 3 shows that cascading two layers exhibit much wider bandwidth. Table 3: Comparison of BW from number of layers point of view

\begin{tabular}{|c|c|c|}
\hline Simulation method & Single layer & Double layer \\
\hline TLM & $18 \%$ & $25 \%$ \\
\hline MOM & $19.8 \%$ & $27.9 \%$ \\
\hline
\end{tabular}

The fabricated structures used RT-Duroid 5880 substrate with height $1.58 \mathrm{~mm}$, and measured using HP 8510c vector network analyzer. The responses with and without the FSS structure were measured and a free space transmission loss (about $5 \mathrm{~dB}$ ) was compensated.

\section{IV- CONCLUSION:}

This work investigated the limit of relative discretization length in the 3D-SCN TLM. The new frontier showed much more accurate results. The validation of this frontier is verified by comparing the FSS response at this limit with the responses of the MoM and the measured data, where perfect matching was achieved. Higher relative lengths showed a noticeable shift in the resonance frequency. This frontier showed a great flexibility, sensitivity, accuracy and versatility when used to simulate the response of the FSS structures by changing the element or substrate properties.

\section{REFERENCES:}

1- P. B. Johns, "A symmetrical condensed node for the TLM-method," IEEE Trans. Microwave Theory Tech., VOL MTT-35, pp. 370-377, Apr. 1987.

2- B. A. Munk, Frequency Selective Surfaces: Theory and Design, John Wiley \& Sons, New York, 2000.

3- ANSOFT DESIGNER software user guide, www.ansoft.com, Version 2.

4- W. J. R. Hoefer, "The transmission line matrix (TLM) Method," T. Itoh, Ed. Numerical Techniques for Microwave and Millimeter Wave Passive Structures. New York: Wiley 1989, pp. 496-591.

5- Mats Gustafsson, Anders Karlsson, Antonio Pedro Pontes Rebelo and Bjorn Widenberg, "Design of Frequency Selective Window for improved indoor outdoor communication", Lund institute of Technology Project review, Sweden, feb.2005.

6- C. Mias, C. Taskonas, C. Oswald, "An investigation into the feasibility of designing Frequency Selective Windows employing periodic structures", The Notteingham Trent University Project review, England, 2002. 
7- Benjamin Hoobetman, "Everything you wanted to know about Frequency Selective Surface Filter but ere afraid to ask", May 2005.

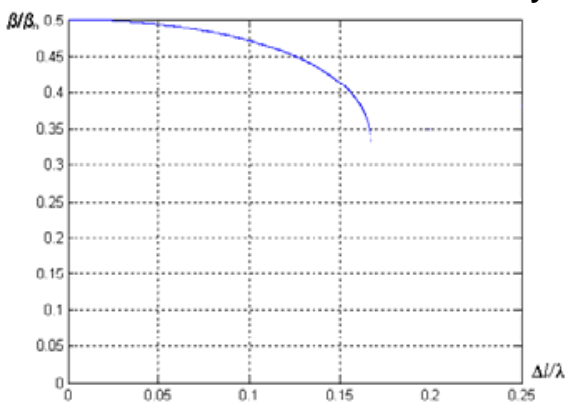

Fig.1: The relation of relative phase constant between TLM network and free space
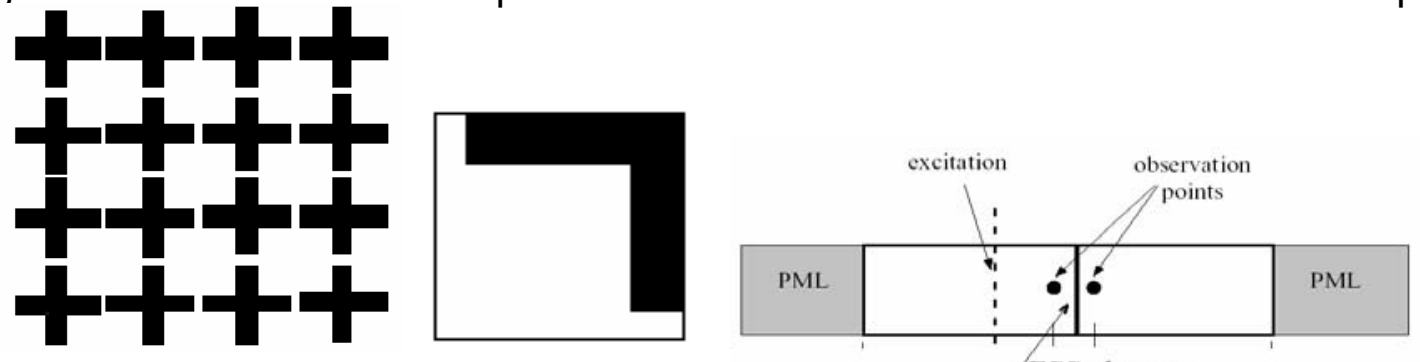

Fig.2: a) Infinite cross dipole FSS structure b) $1 / 4$ of an element with perfect conducting walls to simulate infinite structure in TLM c) PML used to ensure wave propagation along the FSS structure in TLM

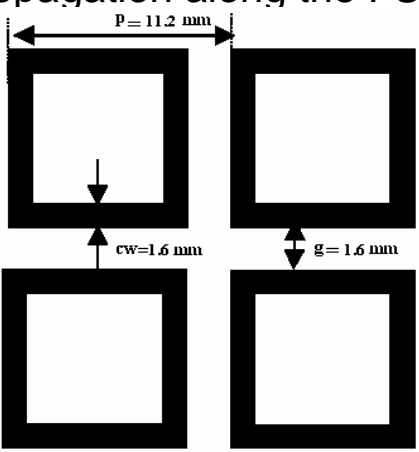

(a)

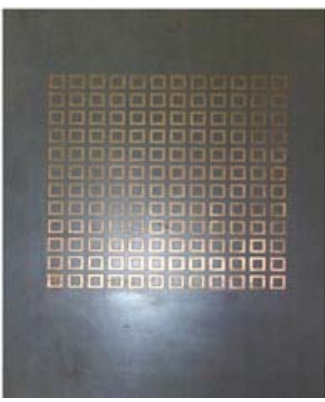

(b)

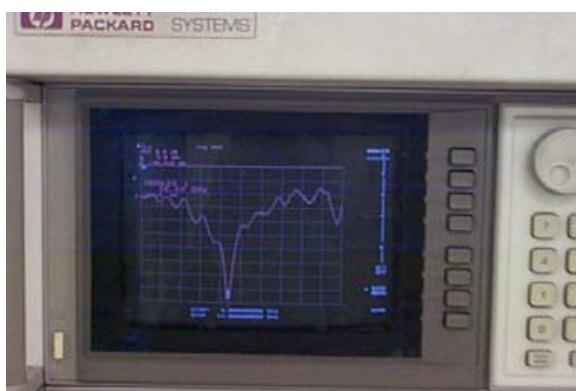

(c)

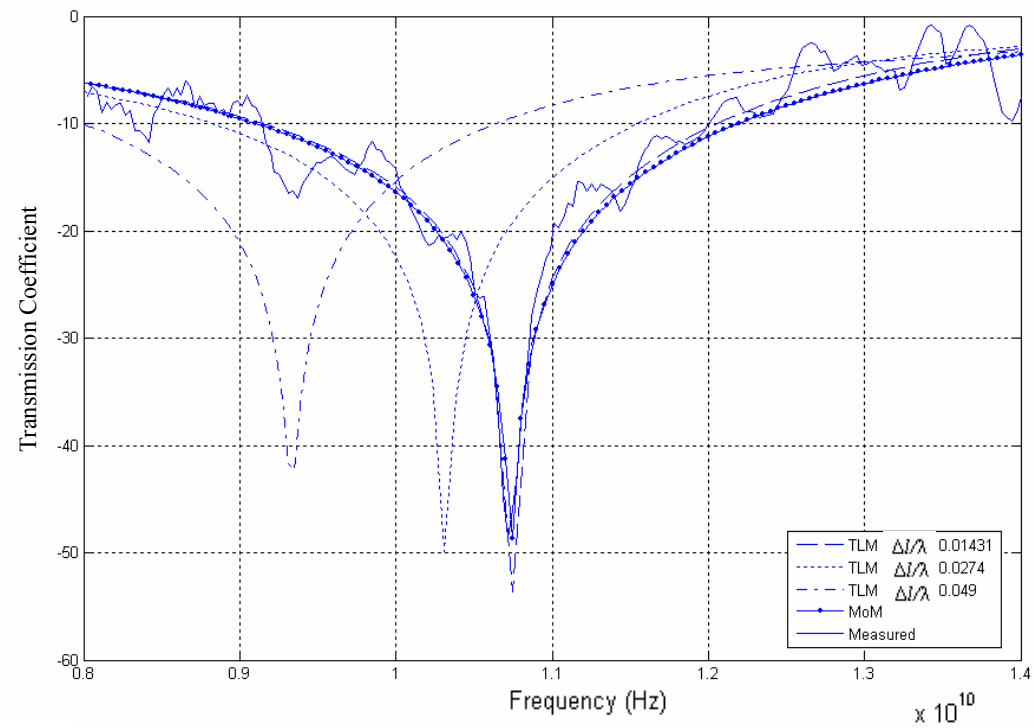

(d) 
Fig.3: a) Rectangular loop FSS element b) The fabricated structure c) Measured data as seen on the network analyzer d) Comparison between TLM, Mom and measured data for transmiss.

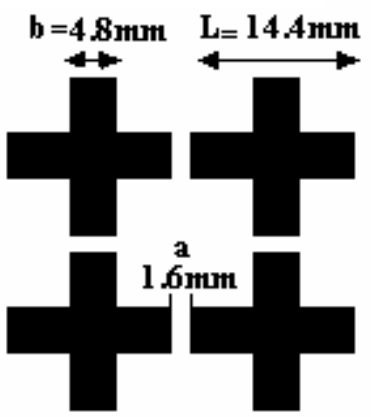

(a)

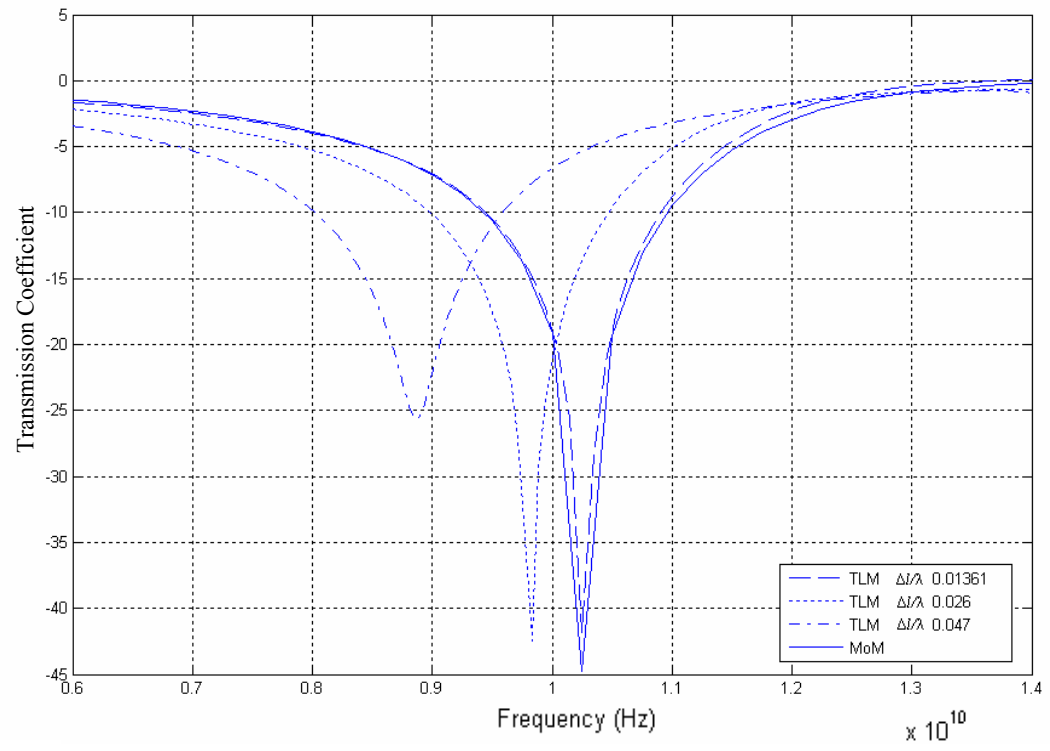

(b)

Fig.4: a) Cross dipole element b) Comparison for simulating cross dipole FSS
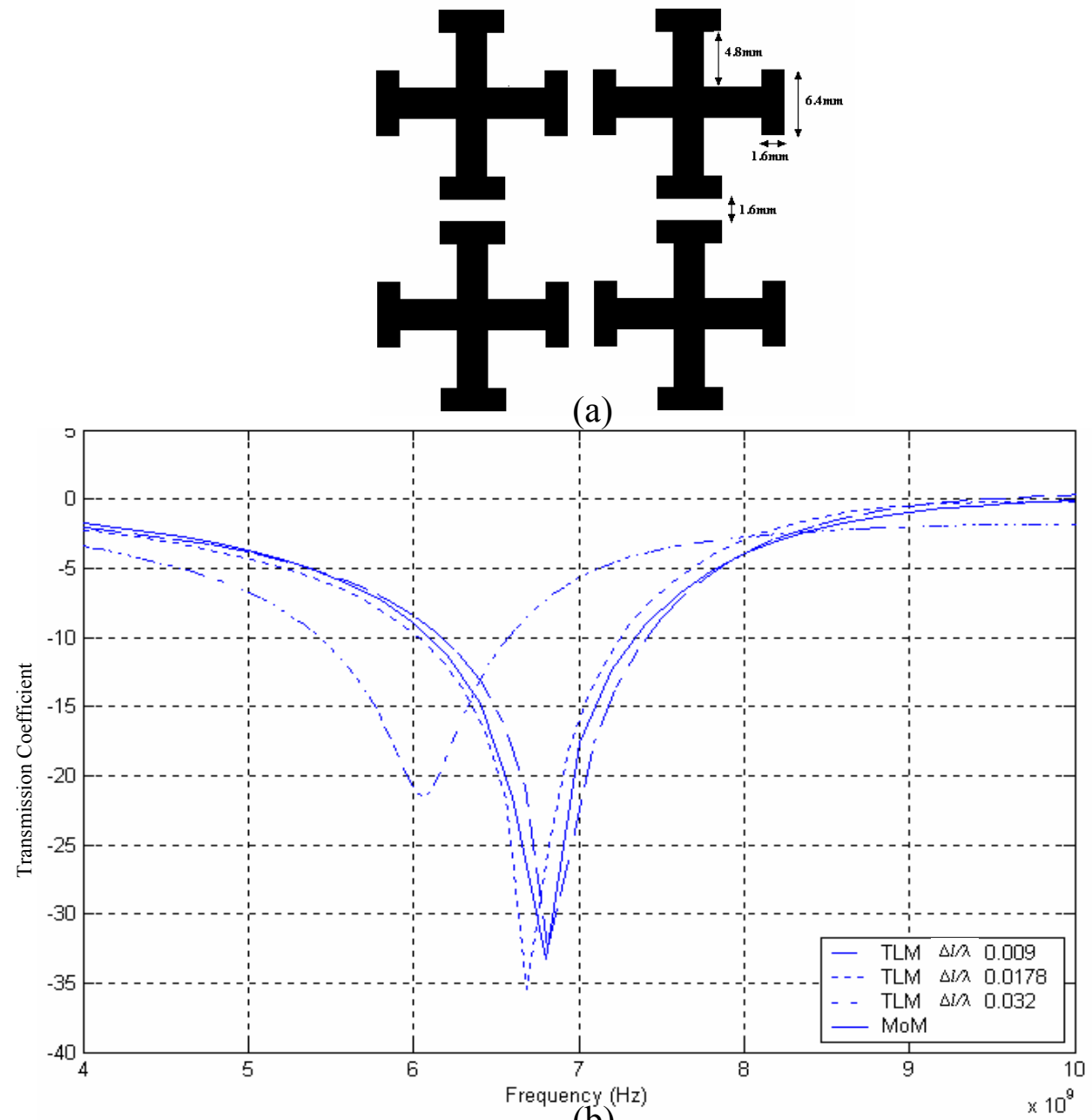

(b) 
Fig.5: a) Jerusalem Cross dipole element b) Comparison for simulating FSS element

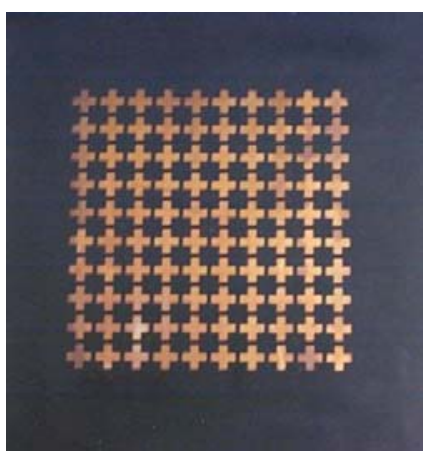

(a)

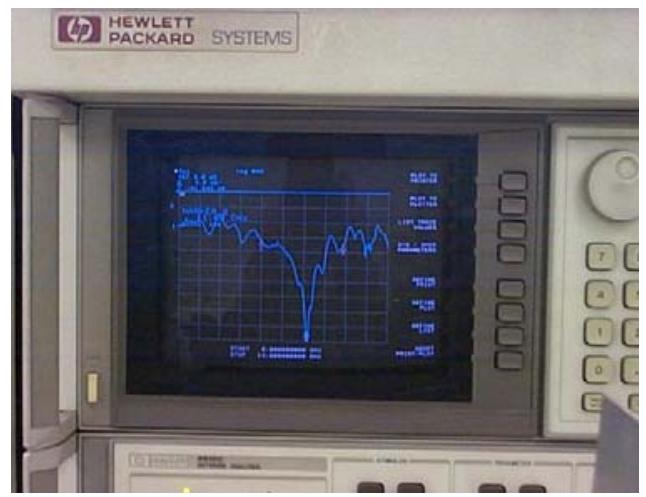

(b)

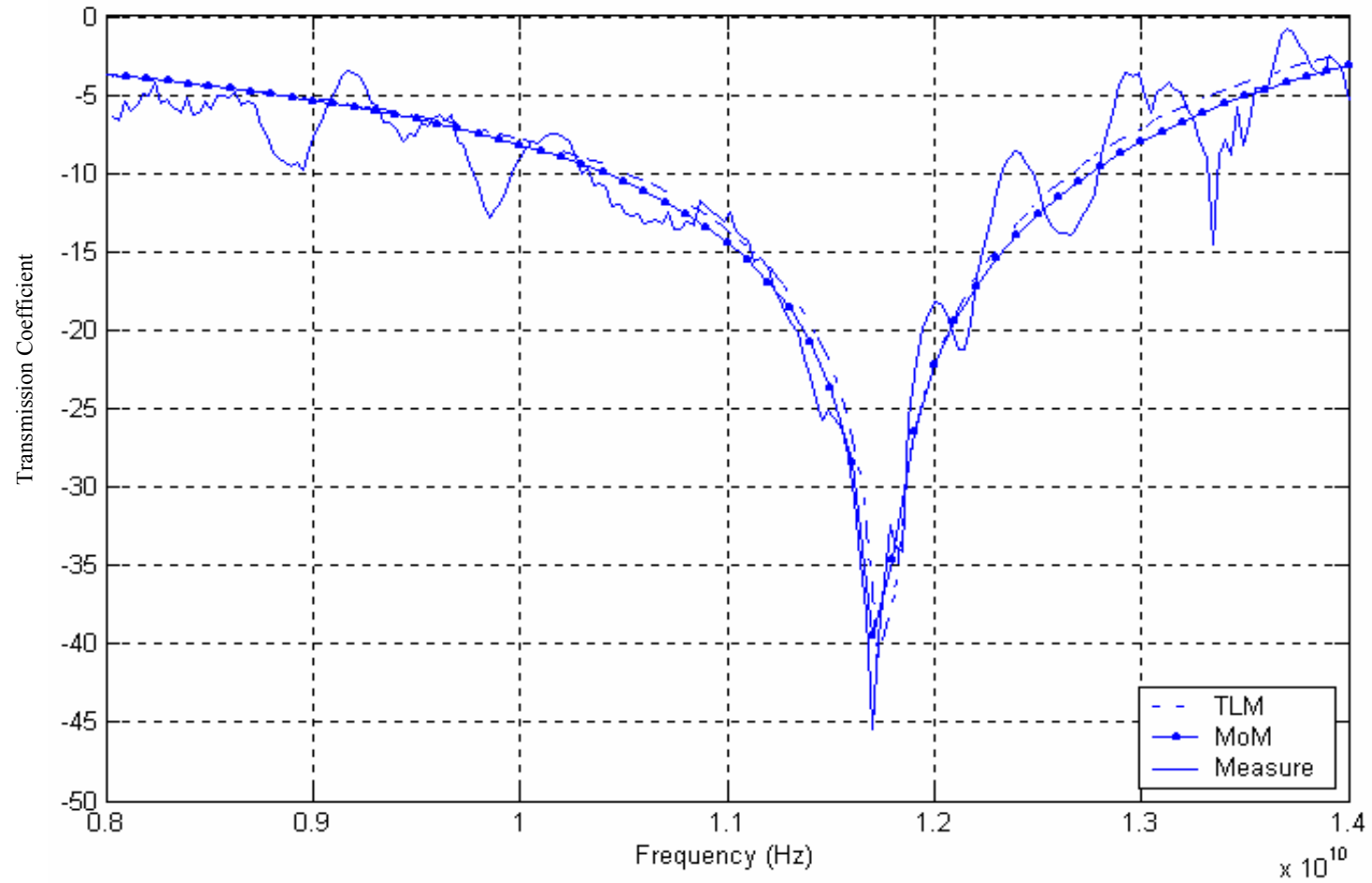

(c) 
Fig.6: a) Fabricated cross dipole structure b) Measured data as seen on the network analyzer c) Comparison between TLM, Mom and measured data

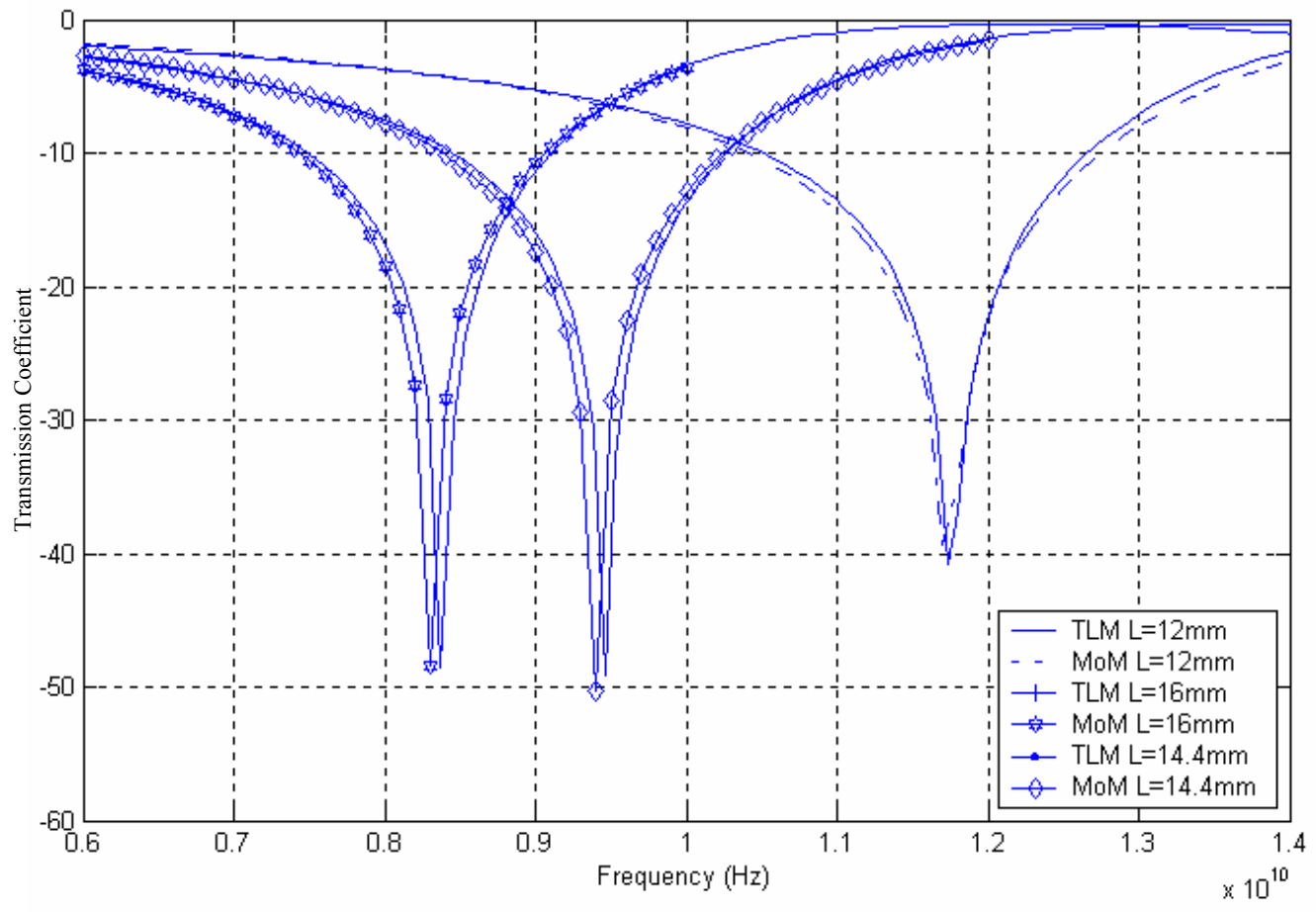

Fig.7: Transmission coefficient of TLM and MoM for changing lengths

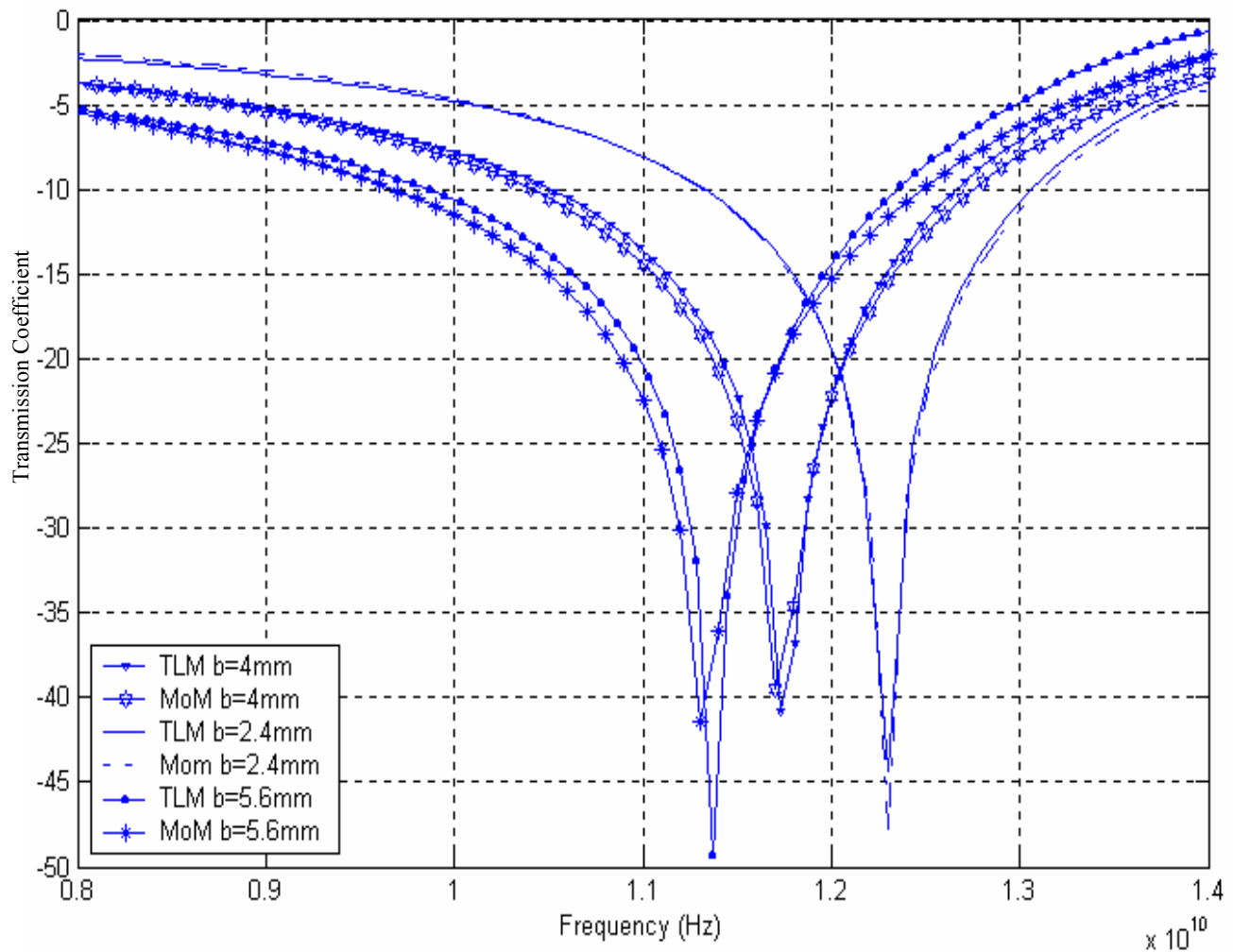

Fig.8: Bandwidths comparison when changing element width 


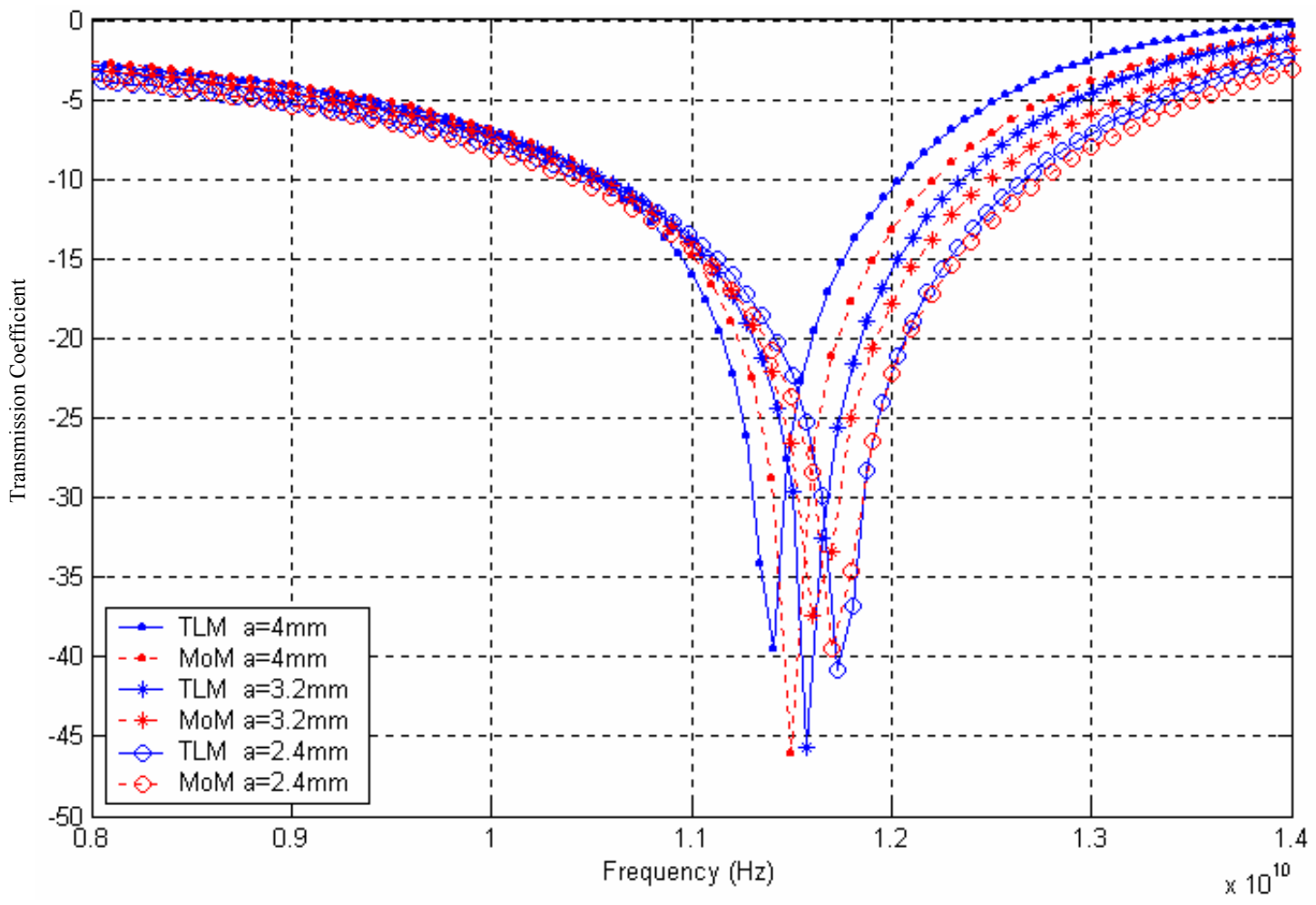

Fig.9: Bandwidths comparison when changing elements inter spacing

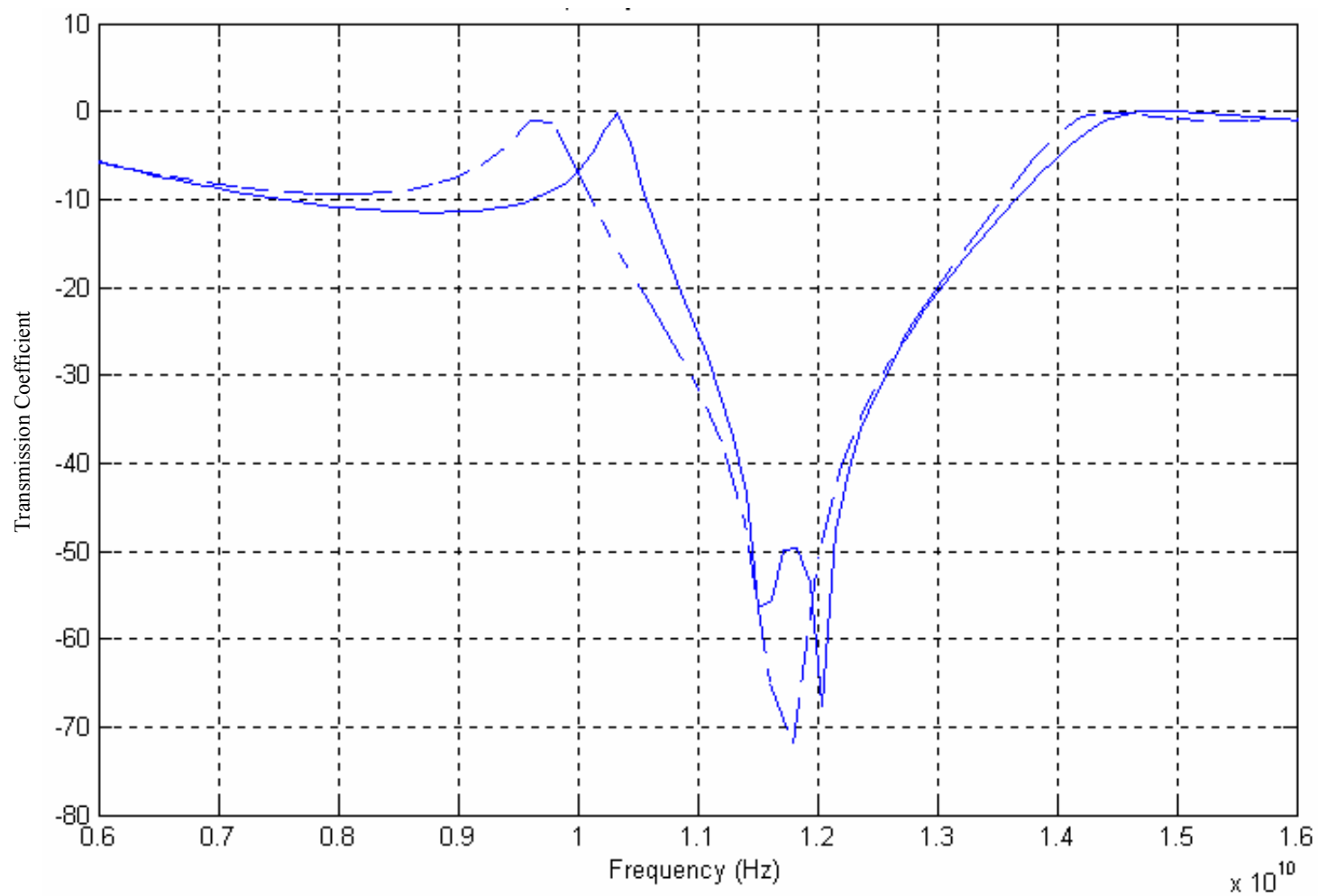


Fig. 10: Comparison of TLM transmission coefficient with MoM for double layer structure (spacing 16mm between layers) TLM (solid line) and MoM (dashed line) 